\title{
Combined TDLAS and OES technique for CO concentration measurement in shock-heated Martian atmosphere
}

\author{
Lin Xin $^{\text {ab }}$, Yu Xilong ${ }^{* b}$, LiFei $^{\mathrm{b}}$, Zhang Shaohua ${ }^{\mathrm{b}}$, Xin Jianguo ${ }^{\mathrm{a}}$, Chang Xinyu ${ }^{\mathrm{b}}$ \\ ${ }^{a}$ School of Optoelectronics, Beijing Institute of Technology, Beijing 100081, China; ${ }^{b}$ State Key \\ Laboratory of High Temperature Gas Dynamics, Institute of Mechanics, Chinese Academy of \\ Sciences, Beijing 100190, China
}

\begin{abstract}
This paper describes the $\mathrm{CO}$ concentration and gas temperature distribution measurements behind a strong shock wave in the simulated Martian atmosphere by an optical diagnostic system. The strong shock wave $(6.31 \pm 0.11 \mathrm{~km} / \mathrm{s})$ is established in a shock tube driven by combustion of hydrogen and oxygen. The optical diagnostic system consists of two parts: the optical emission spectroscopy (OES) system and the tunable diode laser absorption spectroscopy (TDLAS) system. For OES system, high temporal and spatial resolution experimental spectra of CN violet system $\left(B^{2} \Sigma^{+} \rightarrow X^{2} \Sigma^{+}\right.$, $\Delta v=0$ sequence) have been observed. Rotational and vibrational temperature distribution along the shock wave is inferred through a precise analysis of high-resolution experimental spectra. For TDLAS system, a CO absorption line near $2335.778 \mathrm{~nm}$ is utilized for detecting the $\mathrm{CO}$ concentration using scanned-wavelength direct absorption mode. Combined with these experimental results using OES, CO concentration in the thermal equilibrium region is derived. The detected average $\mathrm{CO}$ concentration is $7.46 \times 10^{12} \mathrm{~cm}^{-3}$ with the average temperature of $7400 \mathrm{~K} \pm 300 \mathrm{~K}$, which corresponds to the center fractional absorption of $2.7 \%$.
\end{abstract}

Keywords: optical emission spectroscopy (OES), tunable diode laser absorption spectroscopy (TDLAS), shock wave, temperature, concentration

\section{INTRODUCTION}

Future exploration missions towards the planet Mars will require further understanding of physical and chemical processes occurring during the probe entry in the upper atmosphere ${ }^{1,2}$. Up to now studies, radiation measurements have been widely investigated through OES in shock-tube experiments, and several thermodynamic models have been established to predict nonequilibrium radiation and kinetic phenomena occurring in the shock layer ${ }^{3-7}$. However, the physical and chemical models still have to be validated. Many questions remain on the processes occurring in the shock layer, especially on the temperature distribution and carbon dioxide dissociation. Mars atmosphere is mainly composed by $\mathrm{CO}_{2}(97 \%)$, furthermore, CFD simulations as well as experiments performed in shock tube have shown that one of the most important reactions is the dissociation of $\mathrm{CO}_{2}$,

$$
\mathrm{CO}_{2}+\mathrm{M} \rightarrow \mathrm{CO}+\mathrm{O}+\mathrm{M}
$$

which is the first chemical reaction to occur behind a shock wave ${ }^{8}$. Temperature is one of the most important thermodynamic quantities in shock-induced chemistry because it is a key parameter in chemical reactions. Therefore, accurate quantitative measurement of the concentration of carbon monoxide and the temperature along the strong shock wave will help to study carbon dioxide dissociation and validate the relevant chemical kinetic models $\mathrm{s}^{\text {, } 10}$.

The objective of the present work is to simultaneously measure the $\mathrm{CO}$ concentration and gas temperature distribution behind a strong shock wave using an optical diagnostic system based on the OES and the TDLAS. For the OES system, high temporal and spatial resolution experimental spectra of $\mathrm{CN}$ violet system $\left(B^{2} \Sigma^{+} \rightarrow X^{2} \Sigma^{+}, \Delta v=0\right.$ sequence $)$ are obtained with ICCD gating time as short as 50 ns. The temperature distribution along shock profile can be directly obtained through a precise analysis of high-resolution experimental spectra. The processing method is detailed described in the literature ${ }^{11}$. The TDLAS system is operated in scanned-wavelength direct absorption mode to diagnose one $\mathrm{CO}$ absorption line near $2335.778 \mathrm{~nm}$. Combined with these temperature results using OES, CO concentration in the thermal

\footnotetext{
* Corresponding author: xlyu@imech.ac.cn
} 
equilibrium region, can be obtained by exacting the absorption signal and simple data processing. To our knowledge, this is the first quantitative absorption measurement of $\mathrm{CO}$ concentration at relevant conditions for Mars atmospheric entry.

\section{ABSORPTION SPECTROSCOPY THEORY AND LINE SELECTION}

The fundamental theory of direct-absorption spectroscopy has been detailed by numerous researchers ${ }^{12-14}$, and is reproduced here briefly to define units and terms. The fractional absorption of the laser intensity $\Delta I / I_{0}$ is related to the absorption coefficient $k_{v}$ by the Beer-Lambert law expressed as

$$
\left(\frac{\Delta I}{I_{0}}\right)_{L A S}=\frac{I_{0}-I_{t}}{I_{0}}=1-\exp \left(-k_{v} L\right)
$$

Where $I_{0}$ and $I_{t}$ are the incident and transmitted laser intensities; $L[\mathrm{~cm}]$ is the absorbing pass length; $k_{v}\left[\mathrm{~cm}^{-1}\right]$ is the spectral absorption coefficient. For an isolated absorption transition, the spectral absorption coefficient $k_{v}$ can be expressed as

$$
k_{v}=P_{C O} \cdot S(T) \cdot \phi(v)
$$

Where $P_{C O}[\mathrm{~atm}]$ is the partial pressure of absorbing species, $S(T)\left[\mathrm{cm}^{-2} \mathrm{~atm}^{-1}\right]$ and $\phi(v)[\mathrm{cm}]$ are the line strength and line-shape function, respectively. The product $k_{v} L$ is known as the spectral absorbance $\alpha_{v}$

$$
\alpha_{v} \equiv-\ln \left(\frac{\Delta I}{I_{0}}\right)=k_{v} L=P_{c o} S(T) \phi(v) L
$$

The line-shape function $\phi(v)$ is usually approximated using a Voigt profile which is a convolution of the Gaussian and Lorentz profiles that represent two significant broadening mechanisms. The line-shape function $\phi(v)$ is normalized to unity (defined as $\int \phi(v) d v \equiv 1$ ), so if the temperature $T[\mathrm{~K}]$ is acquired and the wavelength scans strategy is performed, by integrating absorbance over the whole wavelength range, absorbing species partial pressure $P_{C O}$ can be found

$$
P_{C O}=\frac{\int \alpha_{v} d v}{S(T) L}
$$

Based on the ideal gas equation, we can obtain the concentration of absorbers $n_{i}$, which is related to $T$ as

$$
n_{i}=\frac{6.02 \times 10^{23} \cdot P_{C O}}{R \cdot T} m^{-3}=\frac{6.02 \times 10^{17} \cdot \int \alpha_{v} d v}{R \cdot T \cdot S(T) \cdot L} \mathrm{~cm}^{-3}
$$

Here, $R[\mathrm{~J} /(\mathrm{mol} \cdot \mathrm{K})]$ is the gas constant. The line strength at any temperature, $S(T)$, can be calculated from the known line strength at some reference temperature, $S\left(T_{0}\right)$, using

$$
S(T)=S\left(T_{0}\right) \frac{Q\left(T_{0}\right)}{Q(T)}\left(\frac{T_{0}}{T}\right) \exp \left[-\frac{h c E^{\prime \prime}}{k}\left(\frac{1}{T}-\frac{1}{T_{0}}\right)\right] \frac{\left[1-\exp \left(-\frac{h c v_{0}}{k T}\right)\right]}{\left[1-\exp \left(-\frac{h c v_{0}}{k T_{0}}\right)\right]}
$$

where $h[\mathrm{~J} \cdot \mathrm{s}]$ is Planck's constant, $c[\mathrm{~cm} / \mathrm{s}]$ is the speed of light, $k[\mathrm{~J} / \mathrm{K}]$ is Boltzmann's constant, the total partition function of the molecular, $Q(T)$, of CO can be calculated and have been reported in the literature ${ }^{15,16}$, and $E^{\prime \prime}\left[\mathrm{cm}^{-1}\right]$ is the lower state energy of the transition. Here, we use the $296 \mathrm{~K}$ as the reference temperature, while the corresponding line strength $S(296)$ comes from the HITRAN2008 database.

Before selecting, we should analyze the region of gas flow parameters. In our experiments, the absorption length is fixed at $7.8 \mathrm{~cm}$, pressure after the strong shock wave is about $1.1 \mathrm{~atm}$ by thermodynamic calculations, and temperature in the thermal equilibrium region behind the strong shock wave is $7400 \pm 300 \mathrm{~K}$ by optical emission spectroscopy ${ }^{11}$. 
Absorption spectra based on the HITRAN2008 database near $2.3 \mu \mathrm{m}$ are computed for choosing appropriate CO transitions, as shown in Figure 1. The CO absorption line near $2335.778 \mathrm{~nm}$ is selected because it can be convenient calibrated by the absorption line near $2335.544 \mathrm{~nm}$ which can be easily verified by a wavelength meter and a Fabry-Perot interferometer at room temperature.

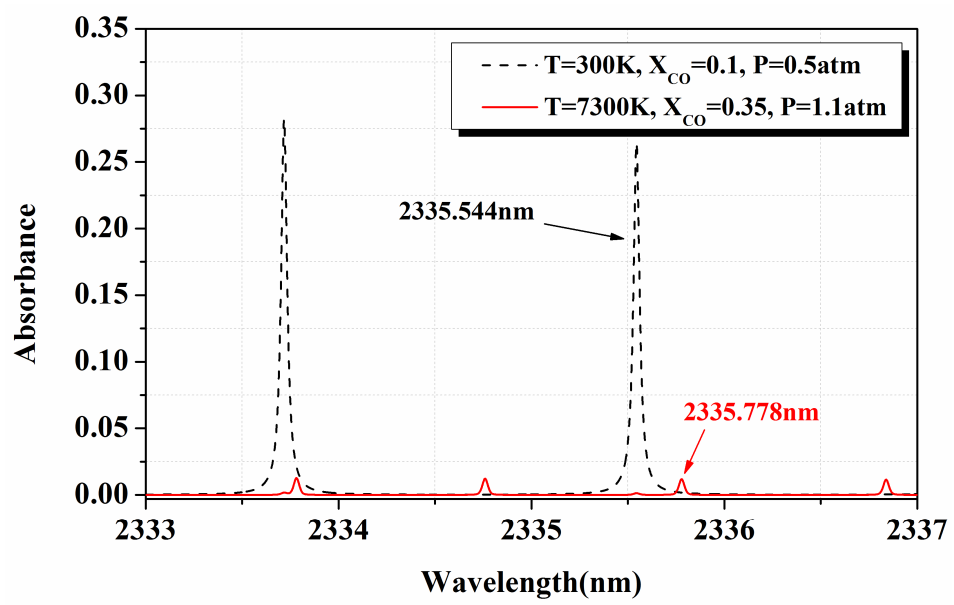

Fig.1 Spectral simulation of $\mathrm{CO}$ absorption near $2.3 \mu \mathrm{m}$ for choosing appropriate $\mathrm{CO}$ transitions

\section{FACILITY AND EXPERIMENTAL SET-UP}

The experiments in this paper are carried out in a hydrogen oxygen combustion shock tube facility. Figure 2 gives an overview of the facility with essential dimensional details and the optical diagnostic system setup. The shock tube is composed of a driver section, an aluminum diaphragm and a driven section. The internal diameter of the stainless steel shock tube is $78 \mathrm{~mm}$. It is equipped with three ion probes that record the shock front during a run and then shock velocity can be measured. Two $10 \mathrm{~mm}$ diameter quartz windows are equipped on both sides of the shock tube to carry out optical measurements. The initial pressures of oxygen and hydrogen in the driver section are $0.06 \mathrm{MPa}$ and 0.59 $\mathrm{MPa}$, respectively. Experiments have been carried out in the mixture constituted of $70 \% \mathrm{CO}_{2}$ and $30 \% \mathrm{~N}_{2}$, despite the difference from the actual composition of the Mars atmosphere, such a ratio allows the $\mathrm{CN}$ radicals emission signal-tonoise (SNR) to be significantly improved whereas the chemical kinetics processes remain the same. The mixture fills the test section with $200 \mathrm{~Pa}$ and the average shock velocity is equal to $6.31 \mathrm{~km} / \mathrm{s}$ with $1.7 \%$ variation.

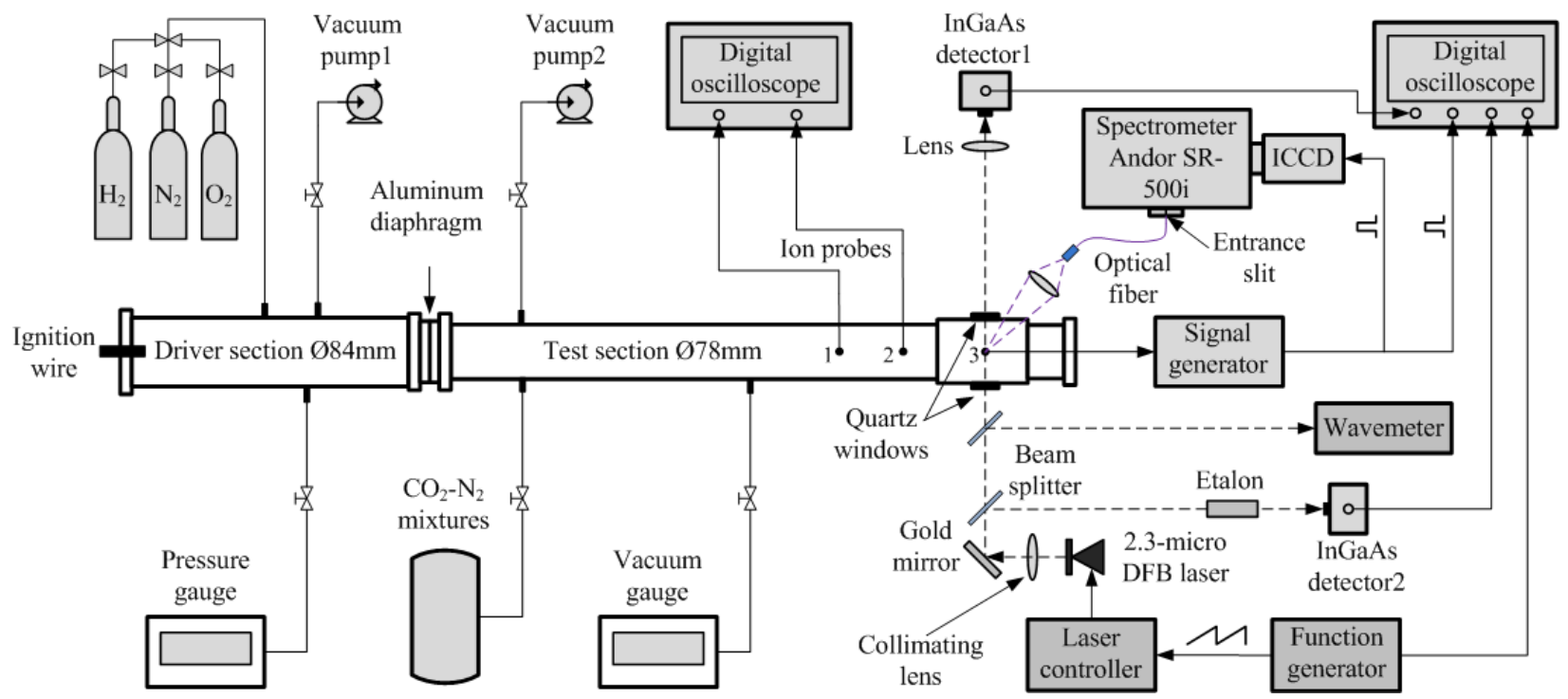

Fig. 2 Schematic diagram of the experimental set-up of the shock tube and the optical instruments arrangement 
The optical diagnostic system consists of two parts: the TDLAS system and the OES system. For the TDLAS system, one distributed-feedback laser (Nanoplus) emitting near $2.3 \mu \mathrm{m}$ is used here. A commercial controller (Thorlabs, Model: ITC-502), which includes temperature and current controllers, drives the diode laser. A function generator (Tektronix, Model: AFG-3101) is used to ramp the laser injection current and thus tune the wavelength of the laser over the desired absorption features. The output from the laser is collimated by an achromatic doublet lens. A gold mirror is used to adjust the beam across the quartz windows on the both sides of the shock tube. Here, a scanned-wavelength direct absorption is used. The laser wavelength is tuned over a range of about $0.7 \mathrm{~nm}$ at a frequency of $50 \mathrm{kHz}$, while the InGaAs detector (Thorlabs, Model: DET10D) signals are recorded by a memory oscilloscope (Tektronix, Model: DPO4034). Using two beam splitters, the two beams of reflected light are simultaneously steered to pass through a Fabry-Perot interferometer (Thorlabs, Model: SA200-18B, 1.5GHz FSR) and a free-space mid-IR wavelength meter (Bristol, Model: 721) for frequency marking.

The OES system arrangement during these experiments is designed to allow the recording of the $\mathrm{CN}$ violet system radiation emitted from the hot test gas immediately following the shock front. The emission has been collected through an optical fiber on which is focused the light coming through a lens system. The role of the lens system is to ensure an acceptable spatial resolution with $1 \mathrm{~mm}$ in diameter and $1 \mathrm{~mm}$ in depth. Spectral information is recorded using an Andor SR-500i $500 \mathrm{~mm}$ focal length imaging, triple grating spectrometer coupled to an Andor DH-740 2048 $\times 512$ pixels gated intensified charge couple device (ICCD). A 1200 grooves $/ \mathrm{mm}$ grating, together with an entrance slit aperture of $100 \mu \mathrm{m}$ are used in the spectrometer giving a spectral resolution of $0.154 \mathrm{~nm}$ (full-width at half-maximum), which is deduced experimentally using a narrow line width diode laser (center wavelength: $777.19 \mathrm{~nm}$, line width $\Delta \lambda<0.001 \mathrm{~nm}$ ).

The optical diagnostic system is triggered by the $3^{\text {rd }}$ ion probe through a signal generator (Stanford Research System, Model: DG535) when the shock wave nears the location. This ion triggering system enabled us to accurately capture the correct flow area of interest. The camera exposure time is set to $50 \mathrm{~ns}$, that means the shock wave only moves about 0.12 $\mathrm{mm}$ which is much smaller than the spectral capture field. Although only one single spectrum can be recorded during a shot, the delay of the gate pulse can be tuned to record different moments in which the spectrum is to be measured in the equilibrium, or in the non-equilibrium region. The acquired spectra are calibrated in relative intensity in the UV range (below $400 \mathrm{~nm}$ ) with a NIST traceable quartz-tungsten-halogen standard light source (Oriel, Model: 63945). The calibration procedure is carried outside the shock tube because of the large size of the tungsten lamp. The signals output from ion probes, InGaAs detectors and the function generator are simultaneously recorded by the Tektronix DPO4034 digital oscilloscope.

\section{RESULTS AND DISCUSSIONS}

\subsection{Rotational and vibrational temperatures determination}

In our studies, $\mathrm{CN}$ is a signature of the chemical reaction between $\mathrm{CO}_{2}$ and $\mathrm{N}_{2}$, thus, it is feasible and important in estimating the temperature using $\mathrm{CN}$ radicals in the high temperature gas mixture behind a strong shock wave ${ }^{17}$. Timeresolved spectra of the $\Delta v=0$ sequence of the $B^{2} \Sigma^{+} \rightarrow X^{2} \Sigma^{+}$electronic transition of $\mathrm{CN}$ have been observed through OES. We analyze in detail the spectral structure of $\mathrm{CN}$ violet system $\left(B^{2} \Sigma^{+} \rightarrow X^{2} \Sigma^{+}, \Delta v=0\right.$ sequence) and propose a rotational method based on fitting high-resolution spectrum. The result of this study shows that the range $(387.6-388.6 \mathrm{~nm})$ of the emission spectra of $\mathrm{CN}$ is controlled only by the rotational temperature. The range of wavelength corresponds to some emission lines of the $\mathrm{P}$ branch (rotational number $J$ range from 0 - 57). According to the measured spectral resolution, rotational temperature can be directly obtained through a precise analysis between an experimental spectrum and theoretical spectrum in this wavelength range. More details and error analysis description of this method are given in the literature $^{11}$. Then the vibrational temperature is estimated by minimizing the difference between the experimental and theoretical spectrum. In order to describe the measurement accuracy, we use the quantity $E r$ to assess the degree of difference between the experimental and calculated spectrum ${ }^{18}$.

All the experimental spectra are compared with calculations to estimate the rotational and vibrational temperatures. Figure 3 presents one representative comparison between the theoretical and experimental spectra when thermal equilibrium is assumed to be achieved. The rotational temperature and vibrational temperatures of the $\mathrm{CN}$ radical are obtained by the proposed method. The profile in the solid line is the experimental spectrum of the $\mathrm{CN}$ violet system and the exposure time of the spectrometer acquiring system is $50 \mathrm{~ns}$. The profile in the dotted line in Figure 3 represents the corresponding theoretical synthetic spectrum with the best-fit rotational and vibrational temperatures. The $E r$ on the top 
panel of Figure 3 indicate that excellent agreement between the experimentally measured and the theoretical synthetic spectrum is obtained and that reasonable rotational and vibrational temperature fits have been achieved.
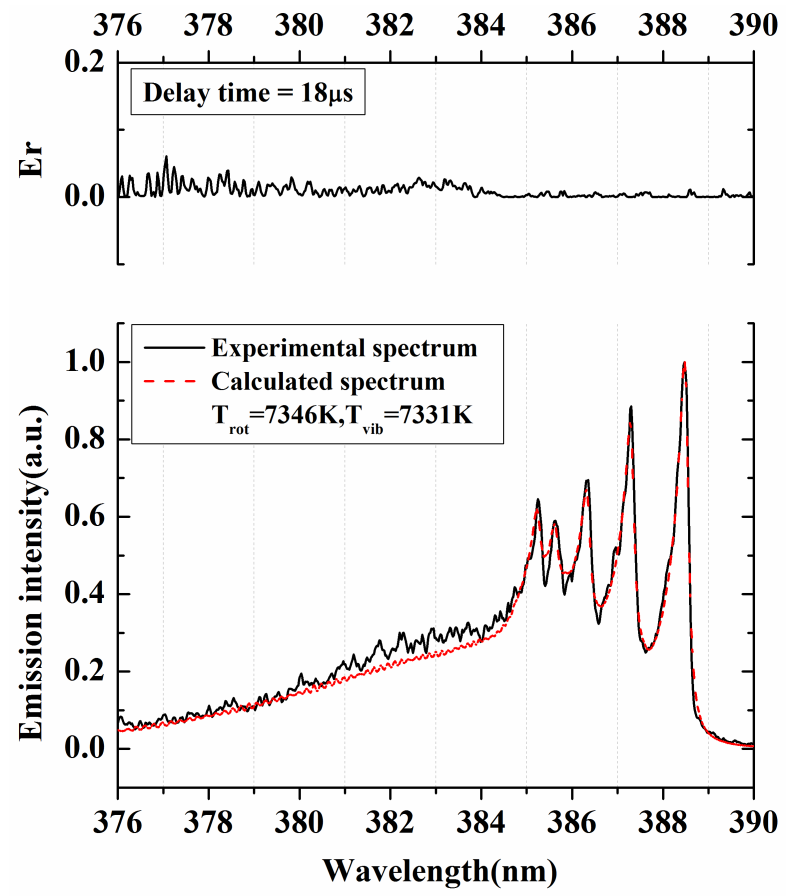

Fig. 3 Experimental and theoretical synthetic spectra of the $\mathrm{CN}$ violet system $\left(B^{2} \Sigma^{+} \rightarrow X^{2} \Sigma^{+}, \Delta v=0\right)$. (Experiment: $\mathrm{P}_{1}=$ $200 \mathrm{~Pa}, \mathrm{~V}_{\text {shock }}=6.31 \pm 0.11 \mathrm{~km} / \mathrm{s}$. Delay time $=18 \mu \mathrm{s}$. Composition: $70 \% \mathrm{CO}_{2}$ and $30 \% \mathrm{~N}_{2}$.)

Figure 4 presents the evolutions of vibratonal and rotational temperatures along the shock profile using OES. The CN emission spectra have been obtained each $200 \mathrm{~ns}$ interval when the medium is considered out of equilibrium and each 2 $\mu$ s when equilibrium is assumed. The temperature decrease behind the shock front is clear and with a relatively large uncertainty on the temperature in the non-equilibrium region. Otherwise, the good agreement between rotational temperature and vibrational temperature after $16 \mu$ s corresponds to an equilibrium temperature $T_{e q}=7400 \mathrm{~K} \pm 300 \mathrm{~K}$.

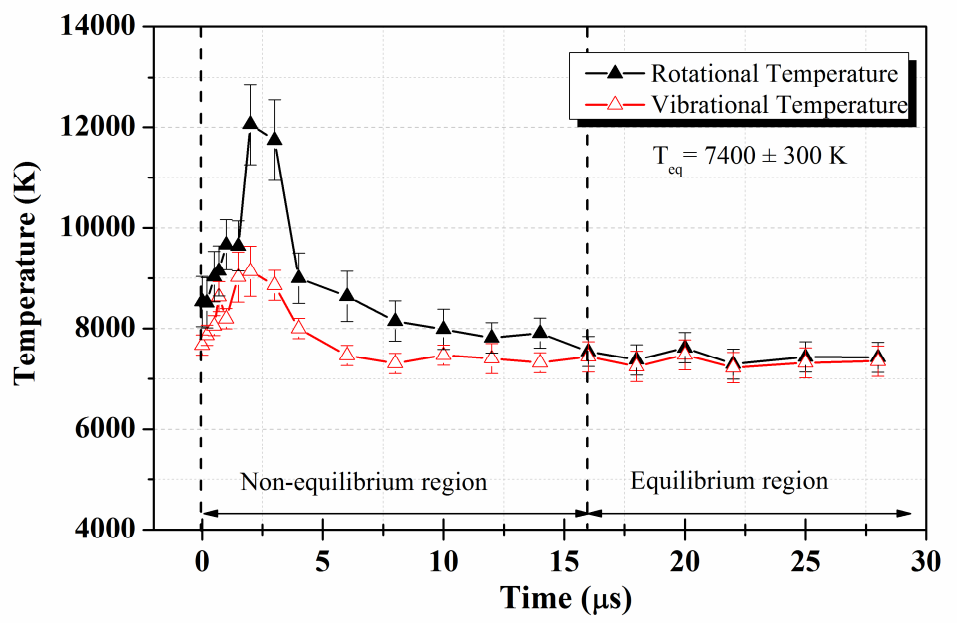

Fig. 4 Evolution of the rotational and vibrational temperatures behind the shock wave (Experiment: $\mathrm{P}_{1}=200 \mathrm{~Pa}$, $\mathrm{V}_{\text {shock }}=6.31 \pm 0.11 \mathrm{~km} / \mathrm{s}$. Composition: $70 \% \mathrm{CO}_{2}$ and $30 \% \mathrm{~N}_{2}$.) 


\subsection{CO absorption measurements behind the strong shock wave}

Since the typical test times of interest in our shock-tube experiments are on the order of $100 \mu \mathrm{s}$, so fast time response is needed for TDLAS measurements. The scan rate using a sawtooth waveform signal $(50 \mathrm{kHz})$ is chosen as a tradeoff between acquiring enough data points per scan with sampling resolution and generating enough scans per test in the 100 $\mu$ s run duration. Although the time to cover the absorption feature is about $1.6 \mu \mathrm{s}$, the temporal resolution of absorption measurement is sufficient for the diagnosis in the equilibrium region because whose effective time is at least $15 \mu$ s by the OES.

Figure 5 shows a representative absorption measurement signal behind a strong shock wave during one shot. In Figure 5 (a), a sharp rising voltage output of the No. 3 ion probe is evident, indication the arrival of the shock at the station of No. 3 probe location and propagates to the downstream region of the tube. Figure 5(b) presents the corresponding etalon signal versus time, which can be used to check the process of changing the absorption scans in time to wavenumber. According to the previous disscussion, the the shock heated gas should reach thermal equlibrium after $16 \mu$ s and the equilibrium temperature is equal to $7400 \mathrm{~K} \pm 300 \mathrm{~K}$, therefore the $\mathrm{CO}$ concentration can be calculated directly by means of the integrated absorbance, using Equation (6). The data processing procedure is usually following this sequence: first, for each measured absorbance signal as shown in Figure 5(c), the non-absorbing wings are extracted and fitted into a polynomial, which is used as the baseline for constructing the absorbance plot of the absorption feature. The baseline polynomial order is adjusted until the baseline absorption signal is zero without any negative values. Here, the baseline is fitted with a fifth-order polynomial. Second, a Voigt profile is used to fit the absorbance plot in the first step. In addition, the chosen baseline also needs to yield a low residual between experiment and the Voigt fit data. The top panel of Figure 6 shows an measured absorption line shape fitted with the Voigt profile, and the bottom portion shows the fitting residual, which is less than $1 \%$ of the peak absorption throughout the line profile. Such low fitting-residual illustrates the high SNR (Signal-to-Noise Ratio) achieved in this research. Finally, based on the Voigt fit obtained in the second step, the integrated absorbance can be calculated, and then used to calculate the $\mathrm{CO}$ concentration. The deduced average $\mathrm{CO}$ concentration is $7.46 \times 10^{12} \mathrm{~cm}^{-3}$, corresponding to the equilibrium temperature $7400 \mathrm{~K} \pm 300 \mathrm{~K}$.
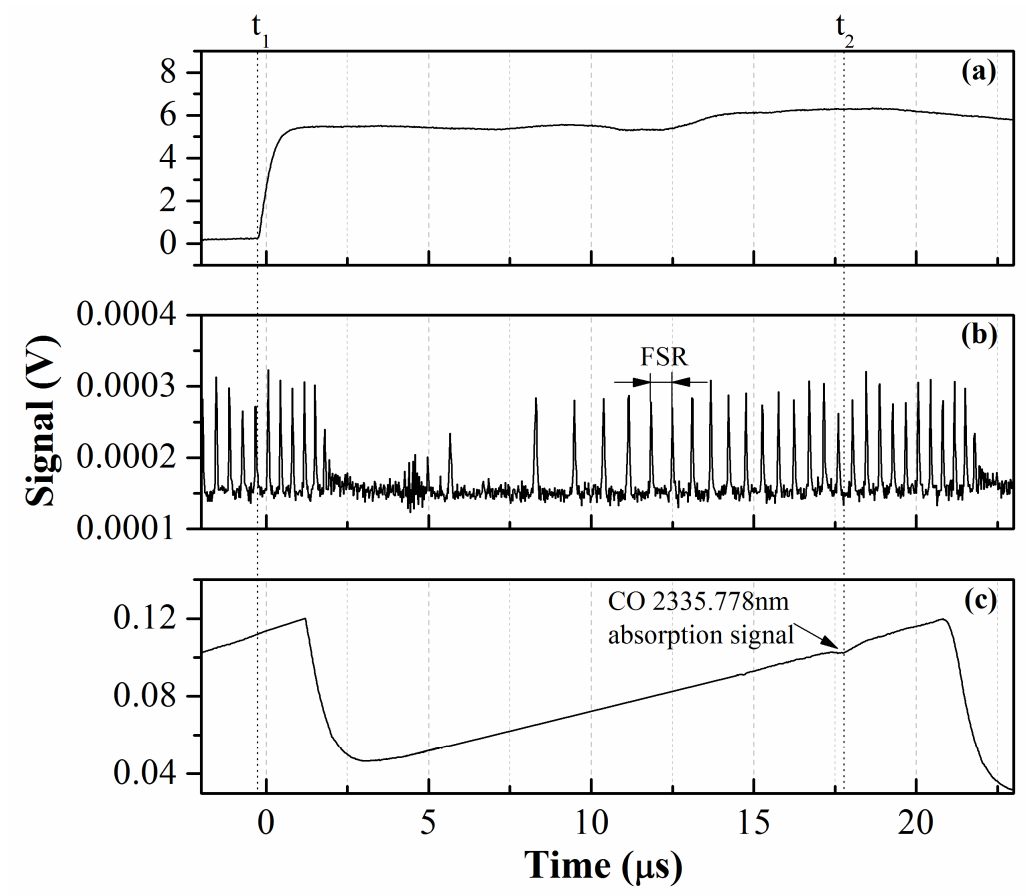

Fig. 5 (a) No. 3 ion probe output versus time, (b) corresponding etalon signal versus time, and (c) corresponding absorption signal versus time under test conditions of $\mathrm{P}_{1}=200 \mathrm{~Pa}$ and $\mathrm{V}_{\text {shock }}=6.36 \mathrm{~km} / \mathrm{s}$ 

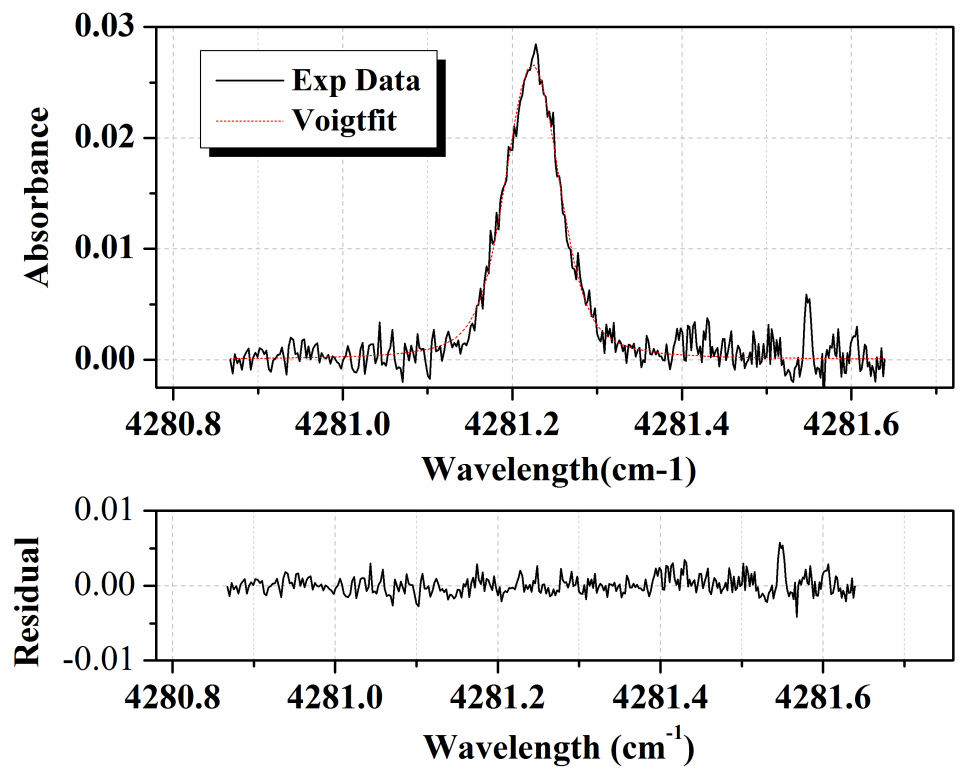

Fig. 6 Single-scan absorption data taken at $50 \mathrm{kHz}$ for concentration measurement in a shock tube with the CO transition near $2335.778 \mathrm{~nm}$. Shown at the top is the best-fit Voigt profile to the experiemental data, while the residual of the fit is shown at the bottom.

Uncertainty in the measure concentration consists of the uncertainty in temperature measurement and uncertainty in the integrated absorbance. As shown in the Equation (6), the uncertainty in temperature ( $4 \%$ ) can also affect the accuracy of line-strength calculation, a $4 \%$ error in the temperature creates a $\sim 5 \%$ the line strength calculation error in our experimental temperature range. The uncertainty in the integrated absorbance is mainly caused by the error associated with baseline and Voigt profile fit. Under our conditions, the high SNR of the baseline and Voigt profile fit, as indicated by the very small fitting residual shown in Figure 7, results in an error of $\sim 1 \%$ in the intergrated absorbance. Therefore, the uncertainty in the measure concetration is about $10 \%$. From the above discussing, we know temperature is the dominant parameter affecting the $\mathrm{CO}$ concentration measurement accuracy.

\section{CONCLUSIONS}

In this paper, experimental results concerning the $\mathrm{CO}$ concentration and gas temperature behind a strong shock wave in Martian-like mixture are presented. The experiments are conducted in a hydrogen oxygen combustion driven shock tube using an optical diagnostic system coupling by OES and TDLAS. High temporal and spatial resolution experimental spectra of $\mathrm{CN}$ violet system $\left(B^{2} \Sigma^{+} \rightarrow X^{2} \Sigma^{+}, \Delta v=0\right.$ sequence) are used to determine the rotational and vibrational temperature distribution along the shock profile. The $\mathrm{CO}$ concentration is measured by a tunable diode laser absorption spectroscopy (TDLAS) system. For this TDLAS system, a CO absorption lines near $2335.778 \mathrm{~nm}$ is selected and analyzed using the HITRAN2008 database. Scanned-wavelength direct absorption mode is utilized at $50 \mathrm{kHz}$ scan rate. The deduced average CO concentration in the thermal equilibrium region is $7.46 \times 10^{12} \mathrm{~cm}^{-3}$, corresponding to the equilibrium temperature $7400 \mathrm{~K} \pm 300 \mathrm{~K}$. An error analysis has been performed to assess the uncertainties from the temperature measurement and the integrated absorbance. As we known, this is the first quantitative absorption measurement of $\mathrm{CO}$ concentration at relevant conditions for Mars atmospheric entry. This results will be very helpful to understand $\mathrm{CO}_{2}$ dissociation and provide verification data for computational fluid dynamic (CFD) simulation in high enthalpy Mars reentry chemical kinetics.

\section{REFERENCES}

[1] Surendra, P. S. and Walter, G. "Nonequilibrium and Equilibrium Shock Front Radiation Measurements," J. Thermophys. Heat Transfer 5(3), 257-265 (1991). 
[2] Braun, R. D., Manning, R. M. "Mars Exploration Entry, Descent, and Landing Challenges," J. Spacecraft Rockets 44(2), 310-323 (2007).

[3] Bose, D., Wright, M. J., Bogdanoff, D. W., et al. "Modeling and Experimental Assessment of CN Radiation Behind a Strong Shock Wave," J. Thermophys. Heat Transfer 20(2), 220-230 (2006).

[4] Boubert, P., Rond, C. "Nonequilibrium Radiation in Shocked Martian Mixtures," J. Thermophys. Heat Transfer 24(1), 40-49 (2010).

[5] Grinstead, J. H., Wright, M. J., Bogdanoff, D. W., et al. "Shock Radiation Measurements for Mars Aerocapture Radiative Heating Analysis," J. Thermophys. Heat Transfer 23(2), 249-255 (2009).

[6] Rond, C., Boubert, P., Félio, J. M., et al. "Radiation Measurements in a Shock Tube for Titan Mixtures," J. Thermophys. Heat Transfer 21(3), 638-646 (2007).

[7] Aaron, M. B., Richard, G. M., Timothy, J. M., et al. "Nonequilibrium Radiation Intensity Measurements in Simulated Titan Atmospheres," J. Thermophys. Heat Transfer 24(2), 291-300 (2010).

[8] Park, C., Howe, J. T., Jaffe, R. L., et al. "Review of Chemical Kinetic Problems of Future NASA mission II: Mars Entries," J. Thermophys. Heat Transfer 8(1), 9-23 (1994).

[9] Rond, C., Bultel, A., Boubert, P., et al. "Spectroscopic Measurements of Nonequilibrium CO2 Plasma in RF Torch," Chem. Phys. 354, 16-26 (2008).

[10] Babou, Y., Rivière, P., Perrin, M. Y., et al. "Spectroscopy Study of Microwave Plasmas of CO2 and CO2-N2 Mixtures at Atmospheric Pressure," Plasma Sources Sci. T. 17, 045010 (2008).

[11]Lin, X., Yu, X. L., Li, F., et al. "Measurements of Non-Equilibrium and Equilibrium Temperature behind a Strong Shock Wave in Simulated Martian Atmosphere," Acta. Mech. Sinica. (Accepted) .

[12] Chao, X., Jeffries, J. B., Hanson, R. K. "Absorption Sensor for CO in Combustion Gases using $2.3 \mu \mathrm{m}$ Tunable Diode Lasers," Meas. Sci. Technol. 20, 115201 (2009).

[13]Farooq, A., Jeffries, J. B., Hanson, R. K. "CO2 Concentration and Temperature Sensor for Combustion Gases using Diode-Laser Absorption near $2.7 \mu \mathrm{m}$," Appl. Phys. B 90, 619-628 (2008).

[14] Meyers, J. M., Fletcher, D. "Diode Laser Absorption Sensor Design and Qualification for CO2 Hypersonic Flows," J. Thermophys. Heat Transfer 25(2), 193-200 (2011).

[15] Goldman, A., Gamache, R. R., Perrin, A., et al. "HITRAN Partition Functions and Weighted TransitionMoments Squared," J. Quant. Spectrosc. Ra. 66, 445-486 (2000).

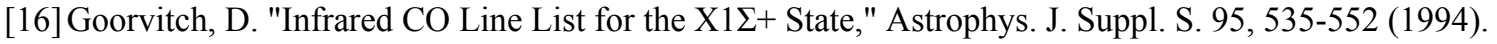

[17] Rond, C., Boubert, P., Félio, J.M., et al. "Nonequilibrium radiation behind a strong shock wave in CO2-N2," Chem. Phys. 340, 93-104 (2007).

[18] Kurosawa, K., Sugita, S., Fujita, K., et al. "Rotational-Temperature Measurements of Chemically Reacting CN Using Band-Tail Spectra," J. Thermophys. Heat Transfer 23(3), 463-472 (2009). 